\title{
Des discours aux pratiques du développement en Afrique : une réflexion sur la problématique du développement local dans le milieu rural Toura (Côte d'Ivoire')
}

\author{
Moustapha Soumahoro*
}

\section{Résumé}

Le développement local est la capacité généralisée d’une société à se prendre en charge, ou encore le processus par lequel une population se donne les moyens de mobiliser ses forces productives dans la transformation de son milieu en vue d'améliorer les conditions de vie et le bien-être de ses membres. De ce fait, le discours sur le développement local tente d'épouser une certaine forme d’homogénéité sur les approches épistémologiques, théoriques, méthodologiques et pratiques mais aussi sur les causes, les objectifs et les finalités. Dans la réalité, la pratique du développement local s'avère plus complexe et cet article se propose, suite à des entretiens avec les paysans Toura et les responsables des organisations communautaires et associatives, de faire écho aux expériences de développement local qui ont cours dans le pays Toura. Dans une telle perspective, l'accent est mis sur la réflexion portant sur le développement local, sur une connaissance de la région Toura, sur le contexte socio-économique afin de fixer les conditions d'un véritable développement et de présenter quelques actions de développement susceptibles d'être mises en application et qui s'articulent autour d'un ajustement des normes, des pratiques et des structures traditionnelles au profit de la « modernité ».

\section{Abstract}

Local development may be defined as the capacity of a society to deal with current issues at hand. It may also be referred to as the process by which a population acquires the means of mobilizing its productive forces in transform-

* Professeur adjoint, Département de géographie, Université Laurentienne, Chemin du Lac Ramsey, Sudbury, Ontario, P3E 2C6, Canada. E-mail : msoumahoro@laurentienne.ca. Site web : http://geography.laurentian.ca 
ing its environment in order to improve the living conditions of its constituents. Therefore, as discussions arise on local development, some may find coalescence on the epistemological, theoretical, methodological and practical approaches; as well as a resolve on the causes, objectives and final results. In reality, the practice of local development is much more complex, as this article proposes, following discussions with local citizens, community and associative representatives from the Toura area of the Ivory Coast. The goal is to study the feedback from experiments of local development which are in progress in the region of Toura. Emphasis will then be placed on the thought-process of the local development, the knowledge of the Toura area in general, and the socio-economic contexts in order to improve the conditions of the true development. This will in turn present some strategies to be applied which will be articulated around an accommodation of the standards, practices and structures traditional with the profit of modernization.

\section{Introduction}

De nos jours, le slogan « Que les populations se prennent en main » est de plus en plus utilisé aussi bien par les acteurs locaux du développement, par les gouvernements africains que par les organismes internationaux. D'ailleurs, l'étude théorique effectuée par la FAO en 2004 portant sur les approches de développements centrés sur les gens en fait l'élément clé des stratégies futures. Il est porteur d'une nouvelle espérance que les anciens modèles de développement n’ont, hélas, pas pu asseoir après des décennies de pratiques et d'expérimentation un peu partout dans les campagnes et les régions reculées d'Afrique. Aujourd'hui, ce slogan se marie étrangement bien au concept de développement local. Et pourtant, le développement local n'est pas en soi un phénomène nouveau. C'est plutôt, le constat d'échec d'une approche de développement centralisé, de type « top-down » et dirigé de l'extérieur (Belloncle 1982 ; Dupriez 1985 ; Pradervand 1989 ; Éla 1998 ; Soumahoro 2003) qui a favorisé un regain d'intérêt pour le développement local perçu, aujourd'hui, comme une alternative crédible (Banque Mondiale 1996 ; FAO 2004).

Dès lors, l'intérêt pour le développement local a favorisé la réflexion sur des stratégies de développement qui doivent s’appuyer sur un plus grand engagement des acteurs auxquelles elles se destinent. Toutefois, les enseignements qui se dégagent des faits observés, ici et là, donnent lieu à certaines interrogations. Au-delà du slogan « Que les populations se prennent en main », quelle signification pratique donner à ce genre de discours ? En effet sur quelle population doit s'appuyer cette prise en main ? Comment cette prise en main peut se faire ? Quel est l'apport extérieur dans cette prise en main ? Quels sont les moyens dont disposent les populations pour cette prise en main ? L'environnement social, économique et physique permet-il cette prise en main ? 
Du discours sur le développement à sa mise en pratique, les choses ne sont pas aussi simples. En effet, les expériences de développement, un peu partout en Afrique, constituent les preuves évidentes de l'écart qui existe entre les discours et les actions. Dans un tel contexte, poser le problème du développement d'une région est un exercice qui nécessite à la fois une réflexion et une prise de position sur la question même du développement et une connaissance du terrain visé essentielle à une action ajustée à la réalité. Quand un tel exercice doit déboucher sur des recommandations en vue d'une action concrète, la stratégie retenue est sans doute ce qui compte avant tout pour les populations concernées (Bernier 1984 ; Dupriez 1985 ; Olivier de Sardan 1985 ; Ouédraogo 1990 ; Hochet 1995; Soumahoro 2003). Il s'agit là bien sûr d'un volet important de tout projet dont l'objectif est d'induire une dynamique particulière dans une région donnée. Il peut arriver que les liens entre l'action proposée et les démarches théorique et empirique qui la soustendent soient flous ou peu convaincants, mais il peut aussi se trouver que ces liens soient transparents et significatifs. Dans ce dernier cas, 1'examen de la stratégie devrait fournir des indications significatives sur le cheminement dont elle n'est que l'aboutissement (Bernier 1984).

Les enseignements qui se dégagent de certains faits observés dans le milieu rural Toura donnent lieu à des interrogations. Peut-on affirmer, avec certitude, l'existence d'une adéquation entre l'importance qu'occupe le développement local dans les discours et les écrits et ce que révèle l'observation des faits ? C'est pour apporter un début de réponse à cette interrogation que la présente recherche a été entreprise. Elle a pour but de faire écho aux expériences qui se passent dans la région Toura à l'ouest de la Côte d'Ivoire (cf. carte $1 \&$ carte 2 ) et par la même occasion contribuer à la réflexion sur le développement et en particulier sur le développement local. Dans une telle perspective, l'accent sera mis sur la question du statut-quo, de la rupture ou de la mutation dans le processus de développement, sur la question de l'autonomie et sur la connaissance du contexte sociopolitique et économique du développement afin de fixer les conditions d'un véritable développement et enfin présenter la stratégie de développement susceptible d'être mise en application.

\section{Une région marginale, un peuple marginal et traditionnel}

Le peuple Toura appartient au grand groupe mandé qui s'étend de la zone septentrionale sahélienne à la zone méridionale forestière de l'Afrique de l'ouest. Il est assimilé à la composante méridionale du groupe mandé communément appelé mandé sud. Il occupe dans l'ouest de la Côte d'Ivoire, un territoire dont l'agencement à l'architecture spatiale régionale constitue une particularité qui se traduit par une marginalité territoriale. L'excentricité 
du pays Toura constitue un handicap majeur dans l'intégration de l'architecture spatiale nationale. Il est localisé à plus de $600 \mathrm{~km}$ des centres nerveux de l'économie et des décisions politiques. Le pays Toura subit l'effet de la distance et est en retrait des grands axes économiques à cause de l'éloignement des pôles urbains importants et de la déficience des infrastructures de communication. Au niveau régional, l'originalité du relief fait de l'espace Toura un espace marginal. Les difficultés de liaison à l'intérieur du territoire en font un territoire peu accessible et l'arrière-pays constitue une vaste région enclavée. Les liaisons inter-villages sont difficiles. Les pistes secondaires sont d'accès difficiles et parfois impraticables. L'espace Toura est faiblement arrimé à l'espace régional.

\section{Carte 1 : Localisation de la région de Toura}

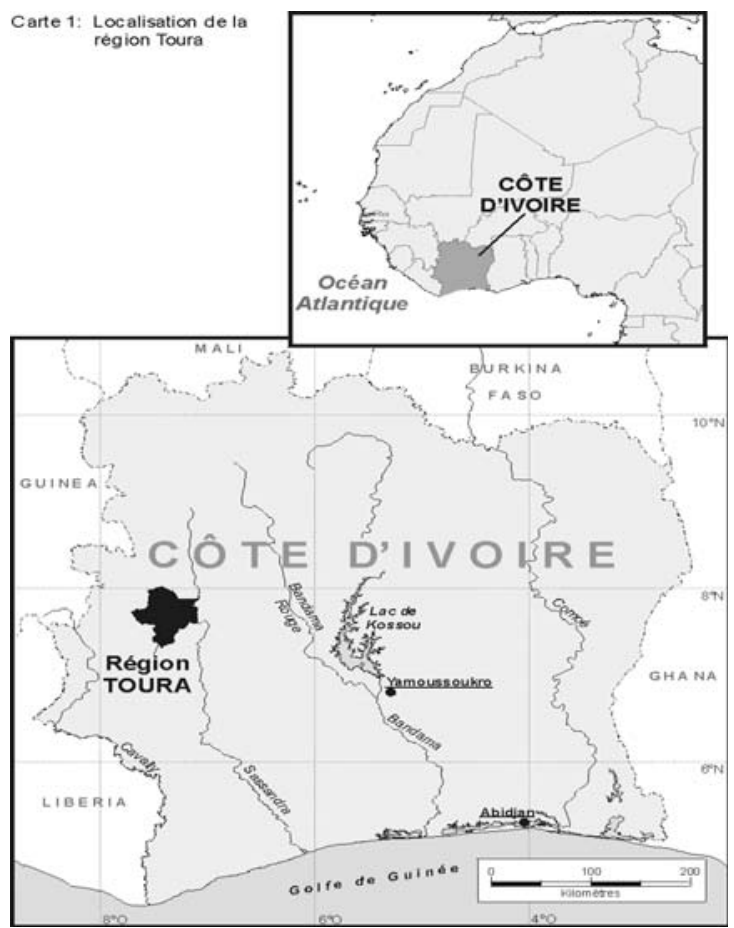




\section{Carte 2 : la région Toura}

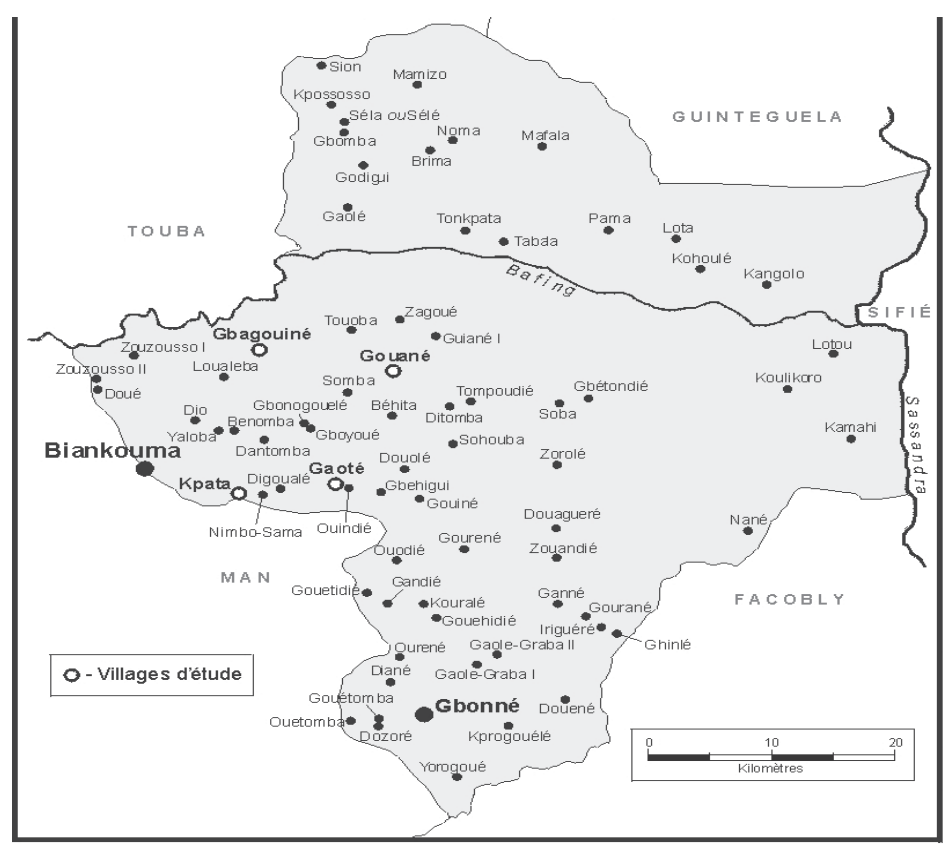

Dans la société Toura, l'individu est un être qui s'accomplit et s'épanouit au travers des balises que la société a fixées. Il évolue dans un cadre familial restreint et étendu au sein duquel il reçoit une éducation et subit les cérémonies initiatiques. La personnalité de l'individu repose sur son éducation en conformité avec les normes sociales et pratiques établies. Le contrôle de l'individu constitue le garant de la cohésion sociale et de la stabilité des institutions traditionnelles. Ce contrôle se traduit par le respect des règles éditées par la société, par le poids qu'exercent les familles, les lignages, les différentes structures sociales.

Le fonctionnement de la famille est hiérarchisé dans la société Toura. Le chef de ménage est responsable d'un noyau familial regroupant sa femme et ses enfants. Le chef de ménage se réfère le plus souvent au chef de famille pour un certain nombre de problèmes à résoudre qui peuvent être liés à son ménage ou qui impliquent le reste de la famille. Il sert de relais entre les chefs de ménage de sa famille et le chef du village.

À l'instar de l'Afrique traditionnelle, l'âge dans la société traditionnelle Toura pose le fondement de l'autorité. La personne âgée a droit au respect et à la considération. De lui-même, l'âge ne donne pas un statut ; mais les relations sociales qui placent les anciens au cœur même de l'organisation et 
de la structuration de la vie en société ont entériné dans la pratique une gérontocratie, responsable exclusive de l'ordre social. L'intérêt que la société porte à ses anciens prend parfois l'aspect d'un culte véritable et le pouvoir que cette gérontocratie détient est essentiellement lié à deux réalités : d'une part à leur capacité d'intervention en matière de « sacré » et d'autre part leur rôle de régulateur des alliances matrimoniales et de la vie familiale au quotidien. L'estime que la population porte aux anciens n'est pas un simple sentiment de gérontophilie, mais elle est renforcée par des sentiments de crainte et d'appréhension du sacré qui constitue un pan important de la vision cosmique du Toura.

La société est très hiérarchisée. Les décisions se prennent au sommet et se diffusent à travers les différents maillons de la société jusqu'à la base. Elles sont du ressort des anciens réunis au sein du conseil du village. Si d'une manière générale, les enfants ne subissent que l'autorité parentale, les jeunes et les femmes par contre subissent l'autorité et les décisions des anciens. Les adultes sont assujettis, dans une moindre mesure, à l'autorité des anciens. Ils constituent le plus souvent les courroies de transmission des décisions des anciens. La femme et les jeunes célibataires restent sous une perpétuelle tutelle.

Le poids d'un certain nombre de structures est encore important dans la société. Qu'il s'agisse des structures sociales, du système matrimonial, de la fête d'initiation, des interdits, du pouvoir des anciens et de leur contrôle sur les femmes et sur les jeunes, il faut avoir à l'esprit qu'ils pèsent dans le fonctionnement actuel de la société et lui confèrent une spécificité qui fait de la société Toura une société originale.

L'agriculture Toura évolue sur un mode de production traditionnel. Les techniques et les pratiques agricoles sont encore rudimentaires. Les exigences d'une plus grande production agricole pour améliorer le revenu paysan et satisfaire les nouveaux besoins se heurtent à des pratiques agricoles peu productives. Les difficultés de développement que connaît le pays Toura s'expliquent par la faiblesse de son agriculture mais aussi par les difficultés à intégrer l'agriculture de subsistance dans un circuit commercial viable d'autant plus que les contrecoups de l'agriculture de rente (café-cacao) laisse le paysan Toura totalement dépendant des spéculateurs et d'un État prédateur.

\section{Statu quo, rupture ou mutation dans le processus de développement ?}

Développer sans abîmer.

Ledéa Ouédraogo 1990.

L'Afrique rurale, à quelques exceptions près, reste encore majoritairement une Afrique des communautés, une Afrique des villages (Belloncle 1982), 
encore attachée à des valeurs et pratiques ancestrales (Belloncle 1982 ; Dupriez 1985 ; Éla 1998) de fraternité de travail, d'entraide familiale, de solidarité communautaire, de cohésion villageoises et de destin commun. Dans un tel contexte, le développement des communautés rurales en Afrique et particulièrement celle du pays Toura nécessite-t-il le maintien et le fonctionnement des anciens modes de production et de gestion sociale ou exige-t-il une rupture ou tout au moins une mutation ?

$\mathrm{Au}$ niveau académique et théorique, la question n'est pas totalement tranchée. Elle a longuement intéressé les chercheurs et inspiré des stratégies de développement, à première vue, souvent divergentes. En effet, deux écoles de pensée s'opposent. Il y a ceux qui pensent qu'il est possible d'adapter les structures traditionnelles à une vie économique plus moderne (Belloncle 1982 ; Bernier 1984 ; Dupriez 1985 ; Olivier de Sardan 1985 ; Ouédraogo 1990 ; Hochet 1995 ; Soumahoro 2003) et ceux qui pensent que la modernisation implique nécessairement un bouleversement, une dénaturation des structures traditionnelles et donc que ces structures sont des freins à la modernisation tant qu'elles subsistent intactes (Meister 1982 ; Banque Mondiale et FAO). Deux auteurs, par leurs écrits et prises de positions, qui restent d'actualité, illustrent parfaitement cette querelle d'école. Il s'agit d'Albert Meister et de Guy Belloncle. Le premier, dans son ouvrage intitulé : Participation pour le développement, fonde sa réflexion et son argumentation sur la disparition des structures traditionnelles villageoises comme condition indispensable au développement des communautés rurales. Le second, dans son ouvrage, $L a$ question paysanne en Afrique noire, prend position pour le maintien des structures villageoises qu'il considère comme le fondement du développement en milieu rural.

La philosophie « mesterienne » du développement repose sur le principe que l'initiative individuelle est pratiquement impossible dans le cadre d'une société traditionnelle à cause du contrôle social s'exerçant sur chacun des membres du groupe. Le postulat de Meister est assez clair : le développement ne peut se faire que sur la base des individus. Dans un tel contexte, quelle alternative peut-il avoir dans une société où la communauté passe avant l'individu ? Pour Meister, rien ne sera possible tant que cette société traditionnelle persistera, et en conséquence est bon tout ce qui contribue à sa déstructuration. Et puisque les sociétés traditionnelles s'opposent à l'émergence d'individus «autonomes», la tâche la plus urgente c'est de les briser. Il faut dans ce cas, selon Meister, « favoriser l'émergence d'entrepreneurs », « encourager le développement de l'esprit des affaires», «mettre en place des procédures de sélection aboutissant au recrutement des plus dynamiques, des plus aguerris, des plus productivistes, de tous ceux 
qui ont le goût du profit et de l'enrichissement individuel ». Ce qu'il faut à l'Afrique, c'est un développement «dirigé par les forts, impitoyable, douloureux». C'est "en Afrique qu'il s'agit encore au contraire de créer des inégalités économiques ». Une étape que l’Europe a réussie alors de quel droit l'Afrique prétendrait-elle y échapper ? Cette interrogation de Meister semble être, dans une moindre mesure, aussi celle de Mendras (1991) lorsqu'il dit ceci : «Pour savoir comment faire entrer les paysans du monde entier dans la civilisation industrielle, afin qu'ils puissent se nourrir et prospérer, le meilleur exemple à méditer ne serait-il pas celui des paysans d'Occident qui ont déjà fait le saut ? »

L’approche préconisée par Meister, qui peut paraître excessive, a inspiré de manière explicite ou implicite les politiques de développement en milieu rural de nombreux gouvernements, d'organismes internationaux et des structures d'encadrement et de développement en Afrique au lendemain des indépendances et jusqu'aux années 80. Dans certains pays, les actions gouvernementales ont consisté à saper les bases de l'autorité traditionnelle ou à mettre en place de nouveaux mécanismes de désignation des autorités villageoises en dehors du cadre traditionnel. La " chefferie administrative » d'origine coloniale qui perdure depuis l'indépendance, et qu'il ne faut pas confondre avec les chefferies précoloniales, en est une illustration. Cette situation n'a pas cours dans tous les pays africains, mais les tentatives n'ont pas manqué avec des réussites et des échecs. En Côte d'Ivoire, si la question de l'autorité traditionnelle se pose différemment en fonction des différentes régions du pays, on note cependant une très faible intervention de l'État pour changer la donne traditionnelle. Toutefois, les chefs apparaissent comme les symboles d'une légitimation des valeurs traditionnelles plutôt que comme de véritables partenaires au développement. Ils sont bons à paraître ornés de bijoux et d'autres apparats traditionnels lors de grandes manifestations et de réceptions, ou encore à agir comme auxiliaire de l'administration auprès des populations, mais cela ne fait pas d'eux de vrais partenaires au développement.

Au niveau des organismes d'encadrement et de développement nationaux et internationaux, les discours ont certes progressivement changé pour intégrer la dimension communautaire et participative du développement dans les stratégies, mais, dans la pratique, le degré d'engagement reste encore flou. L'intérêt est toujours porté sur l'initiative individuelle et privée, qui par son dynamisme aura un effet d'entraînement sur le reste de la communauté villageoise et, de ce fait, sur le développement du village. À ce titre, la disparition des structures traditionnelles semble être la seule alternative possible pour un développement des sociétés rurales traditionnelles. Cette position n'est pas partagée par Belloncle (1982) qui pense que non seulement la 
disparition des structures traditionnelles n'est pas la seule alternative, mais qu'elle n'est pas la meilleure solution.

Il propose donc le développement par les communautés contrairement à Meister qui milite pour leurs disparitions au profit de l'individu. Face à une telle divergence, quel doit être le fondement du développement en milieu rural ? Le développement doit-il être quelque chose d'individuel tendant à faire disparaître les structures traditionnelles ? Et si oui, faut-il dans ce cas introduire de «force» l'initiative individuelle par la négation des structures traditionnelles comme le préconise Meister et comme cela fut maintes fois tenté ? Contrairement aux idées largement diffusées dans les ouvrages d'histoire, les formes d'organisation socio-économiques récentes en Afrique et en particulier dans le pays Toura se différencient aisément de celle des communautés primitives. Elles reposent sur un dualisme entre l'individu et le collectif constituant ainsi un socle solide. Parmi les atouts, d'un tel dualisme, il faut retenir des structures de décision consensuelle et une grande homogénéité économique se traduisant, en particulier, par 1'egalité d'accès à la terre et la faible différenciation dans l'accès au moyen de production. Selon Belloncle (1982), seule l'appropriation collective du sol et des formes coopératives de travail que l'Afrique doit à son fond coutumier, peuvent expliquer une telle situation. Pourtant, le maintien de tels éléments n’a pas empêché la campagne africaine d'incorporer des éléments du système capitaliste introduits par le colonisateur, notamment l'initiative de la production revenant au paysan, la culture individuelle des champs et l'appropriation privée de ses fruits, et 1'écoulement sur un marché d'une partie de la production. En pays Toura, l'adaptation s'est faite parfaitement. L'activité agricole de subsistance s'exerce concomitamment avec l'agriculture de rente (café et cacao). Le paysan Toura est propriétaire de sa plantation de café ou de cacao dont il essaie d'écouler la production pour satisfaire ses besoins et ceux de sa famille et de réaliser ses projets.

Évidemment ces nouveaux éléments, qui traduisent l'adaptation du traditionnel et du moderne, peuvent devenir des germes de décomposition du fonds coutumier, s'ils ne bénéficient pas à court terme d'un contexte socioéconomique dont la cohérence contrôlerait leurs effets différenciateurs. Cette situation permettrait, ainsi, une évolution vers le système auquel tend la société moderne sans passer par la destruction des structures traditionnelles. Face à la capacité d'adaptation de la campagne africaine et des paysans, notre intime conviction est que les structures traditionnelles constituent un atout majeur dans le processus de développement du milieu rural africain et en particulier du milieu rural Toura. Il serait illusoire de souhaiter leur disparition afin que le développement se fasse. Elles font partie de la solution et non du problème. 
Il faut nécessairement s’appuyer sur ces structures pour relever les défis du développement. Il suffit dans ce cas de corriger les dysfonctionnements et autres insuffisances des structures traditionnelles ou des pratiques traditionnelles et éviter ainsi de jeter le bébé avec l'eau du bain comme semble le préconiser Meister dans son approche de développement du milieu rural. En conclusion, nous ne sommes pas partisan d'un statut-quo encore moins d'une rupture mais plutôt d'une mutation, d'un processus d'adaptation des structures traditionnelles Toura permettant d'aller vers plus d'autonomie et d'efficacité dans l'action. Les projets initiés depuis les années 90 par les organismes internationaux et surtout les ONG, actives dans les actions de développement local, tentent de favoriser cette approche pragmatique consistant à s'appuyer sur les structures existantes pour incuber le développement. Les études et recherches initiées par certains auteurs (Belloncle 1982 ; Beaudoux 2001 ; Prevost 2001 ; Lévy 2000 ; Éla 1982,1998 ; Aubin 1991 ; Bernier 1984, 1988 ; Dupriez 1985 ; Olivier de Sardan 1985 ; Ouedraogo 1990 ; Hochet 1995 ; Soumahoro 2003) ont souligné la nécessité d'encourager un développement local dans les milieux ruraux africains qui tient compte à la fois des réalités intrinsèques du milieu et les exigences de développement et de renouveau dont sont porteur les politiques initiées par l’État, les organismes internationaux et les ONG.

\section{Autonomie : facteur clé de succès d'un processus de développement au niveau local ?}

Aux différentes politiques d'intervention directe dans le milieu rural des États avec des résultats souvent mitigés s'est substituée progressivement une alternative fondée sur l'idée de la satisfaction des besoins élémentaires des individus dans un contexte d'autonomie totale ou partielle. Cette approche envisage le développement comme un processus fondé d'abord sur une mobilisation des ressources naturelles et humaines de chaque région avec comme objectif premier la satisfaction des besoins de base des habitants de la région. À cette fin, les politiques de développement préconisent des stratégies nécessitant une main-d'œuvre importante, mais peu exigeantes en capitaux, applicables à l'échelle locale, fondées sur les ressources régionales et ayant recours à une technologie appropriée. Cette approche a reçu depuis quelques années une bonne dose de support académique et rhétorique, mais elle a rarement inspiré des stratégies de développement (Bernier 1984) des États qui sont restés attachés à une politique d'intervention directe.

L'observation des politiques de développement agricole et du monde rural de l'État de Côte d'Ivoire permet difficilement d'affirmer avec certitude qu'une stratégie axée sur l'idée de la satisfaction des besoins élémentaires des individus dans un contexte d'autonomie totale ou partielle a été menée 
dans le pays Toura. Même si, au niveau des programmes de développement sectoriel appuyés par des organismes internationaux comme la Banque Africaine de Développement (BAD), dans le cadre du projet BAD Ouest, plusieurs actions initiées laissent entrevoir une telle stratégie dans la région Ouest de la Côte d'Ivoire, sa matérialisation sur le territoire Toura reste très limitée. L'existence de quelques structures décentralisées d'encadrement et d'appui aux paysans est très loin de rentrer dans ce schéma stratégique. Le plus souvent, leur mode d'opération s'inscrit beaucoup plus dans une approche d'intervention directe par la réalisation de projets avec une obligation de résultats que dans la recherche d'un développement nécessairement axé sur l'autonomie des partenaires locaux.

Face à cette réalité, notre conception du développement, basée elle aussi sur l'idée de la satisfaction des besoins essentiels, nous a amené à définir le développement comme la capacité généralisée d'une société de se prendre en charge, ou encore comme le processus par lequel une société se donne les moyens de mobiliser ses forces productives dans la transformation de son milieu en vue d'améliorer les conditions de vie et le bien-être de ses membres. Bref, un processus global incluant 1'ensemble des aspects de la vie (environnement, activités de production et d'échanges, comportement de consommation, culture) et impliquant la participation des collectivités locales tout autant à la prise de décision qu’à la réalisation des activités porteuses de progrès. Ainsi envisagé, le développement réside moins dans un résultat atteint que dans un ensemble d'activités réalisées selon les attentes des collectivités concernées, avec leur accord et leur participation.

C'est cette approche du développement qui a guidé notre démarche de recherche dans les quinze villages visités et surtout dans les quatre villages retenus pour l'étude. L'enquête sur le terrain, basée sur les focus groups avec les responsables villageois et les associations villageoises, l'administration de questionnaires à 120 paysans ainsi que l'observation du finage des différents villages, ont permis de se faire une idée de la question du développement au niveau local. L'enquête a permis d'identifier les problèmes et les besoins des populations et aussi d'entrevoir les approches de solutions initiées en fonction des moyens et des possibilités qu’offre le milieu Toura.

\section{Le contexte socio-politique et économique}

La région Toura connaît une situation de sous-développement qui n'est, somme toute, pas significative jusqu'à présent. Le caractère marginal du territoire a permis la survivance d'un système de production traditionnel voué à sa propre reproduction. Aussi, sous plusieurs aspects, mode de vie, division du travail, formes de solidarité, rythmes de production, etc., il s’agit davantage de problèmes d'adaptation aux contraintes liées au nouvel 
environnement socio-économique introduit par la modernité que d'un véritable sous-développement et une dépendance réelle avec un système économique extérieur. Il s’agit d’une société « secouée ", encore fortement marquée par le passé, mais où se manifestent depuis quelques décennies des mutations réelles qui risquent de s’accélérer.

La société Toura est une société en transition, encore dominée par un système gérontocratique plus préoccupé de préserver l'ordre social traditionnel que d'assurer véritablement le développement local. Elle ne peut, non plus, guère compter sur les interventions d'un État ivoirien dont les capacités de financement ont été considérablement réduites. Si la société Toura se doit d'amorcer son propre développement, il lui faut encore s'appuyer en priorité sur des actions communautaires, collectives, car elle n'a pas encore vu l'émergence d'une classe suffisamment importante de paysans innovateurs susceptibles de l'entraîner vers un développement rapide (Soumahoro 2003). Le peuple Toura s'est intégré tardivement à l'économie marchande et à l'ensemble social ivoirien. Cela signifie que tant sur le plan culturel que social, les étapes du développement qui ont marqué l'ensemble de la Côte d'Ivoire au cours des quatre dernières décennies n’ont pas encore été totalement intégrées (Hauhouot 1998 ; Soumahoro 2003). La gérontocratie demeure importante dans la société Toura. L'organisation sociopolitique de la société Toura montre la forte prégnance des structures traditionnelles. Elle souligne la permanence des rapports traditionnels à la terre et des rapports sociaux selon une hiérarchie qui contraint fortement les initiatives individuelles (Holas 1962 ; HCDI-RSMO 1998 ; Soumahoro 2003). Le paysan doit se référer constamment à sa famille, à son groupe familial, à son lignage ou à l'aîné de la famille pour prendre certaine décision qu'il considère vitale pour son avenir et pour les autres membres de sa famille.

Les conditions de production agricole et les conditions de vie demeurent difficiles. L'excentricité de la région au sein du territoire ivoirien, la dispersion et les difficultés d'accessibilité des villages ainsi que le manque de moyens de l'État ont considérablement affaibli les interventions administratives et gouvernementales. Également, la faible implication des structures locales de pouvoir décisionnel dans le processus de développement ne soutient pas une prise en charge endogène du développement agricole et économique. Celuici est perçu comme ne pouvant être initié et soutenu que par des interventions extérieures, gouvernementales qui ne se manifestent que marginalement par l'encadrement administratif et technique et par l'implication occasionnelle de rares ONG (Soumahoro 2003). Les acteurs individuels sont rares car le pays Toura n'a pas vu jusqu’à présent l'émergence d'une paysannerie innovatrice et engagée dans la transformation du système de production agricole. L'émergence d'une classe de producteurs résolument engagés dans 
l'économie marchande demeure soumise au contrôle social et aux pratiques lignagères et collectivistes (Soumahoro 2003).

\section{Les conditions d'un développement local}

Les efforts de développement entrepris, soit par les populations Toura, soit par les structures gouvernementales, impliquent nécessairement une remise en cause de certaines pratiques traditionnelles de la société. Même si l'aspect d'une évolution en autarcie constitue un gage de sécurité contre une transformation radicale dont tous les contours sont difficilement cernés, il est certain qu'à long terme la société Toura devra se transformer. Le degré de transformation dépendra de la manière d'envisager le développement et des alternatives possibles qui s'offrent aux populations en tant qu'acteurs mais aussi bénéficiaires d'un tel processus. Il s'agit pour la population de trouver de nouveaux points d'appui pour assurer sa continuité tout en permettant une mobilisation effective des forces productives dans l'effort de développement local. Par quels moyens assurer ce changement dans la continuité ? Par la réalisation d'un certain nombre de conditions qui, somme toute, dépendent en partie des facteurs sociopolitique et économique internes à la société Toura.

\section{Préserver les acquis essentiels de la culture Toura}

Comme toute société confrontée à une influence extérieure, la société Toura peut-elle à la fois s'ouvrir au changement, maintenir sa cohésion et préserver les valeurs propres à sa culture ? À première vue, les conditions d'existence et les circonstances actuelles, sans être des atouts réels, lui sont favorables. La marginalisation territoriale et le peu d'ouverture sur l'extérieur ont permis de préserver encore un socle traditionnel dans le respect des valeurs et principes fondateurs de la société. Toutefois, le danger vient de l'introduction de techniques et surtout de nouvelles connaissances et de la scolarisation qui constituent des canaux parallèles de diffusion du savoir autres que celles des anciens et une menace potentielle de l'autorité traditionnelle. L'émergence des responsables d'associations de développement, généralement scolarisés, dans les villages Toura et jouant un rôle de plus en plus important en est la parfaite illustration. Dans ce cas, comment concilier, des changements qui semblent inévitables et la solidarité sociale, socle de la société Toura? L’enquête terrain ne permet pas de répondre avec certitude à cette question. Toutefois, tout n'est pas noir ou blanc. On observe des processus d'adaptation, de recherche d'équilibre entre valeur traditionnelle et les réalités modernes. En effet, il ressort des entretiens avec les paysans Toura qu'ils sont conscients de la nécessité de préserver les acquis de la société traditionnelle et de la nécessité de s’ouvrir sur l'extérieur. Il va de soi que le paysan Toura est 
flexible et il n'est pas aussi réfractaire au changement. Comme exemple, nous avons l'’intégration de la culture de café et cacao (deux cultures de rentes extérieures au milieu Toura) à la culture de subsistance. Ce qui a permis l'acquisition de nouvelles pratiques de travail qui constitue aujourd'hui le socle de l'activité agricole. L'adoption de nouvelles techniques n'est pas rejetée, elle est consentie, même si l'impact d'une telle adoption n'est pas considéré. Cet impact peut être négatif dans la mesure où le milieu social exerce encore un contrôle sur l'individu et l'efficacité de la technique importée peut entraîner un sentiment de défavorisation de la culture pouvant entraîner soit la dépendance ou l'attentisme face à l'aide extérieure. D’où la nécessité d'introduire des changements techniques, à petite dose, et de faire en sorte qu'ils accompagnent les changements sociaux. Ces changements doivent être assumés et mises en place par les communautés. C'est de cette façon qu'un changement souhaité pourra être compris et accepté par tout le monde.

\section{Débarrasser la culture de ses insuffisances}

Il est plus facile de détruire une molécule d'atome que de détruire une coutume. Albert Einstein.

Le discours classique qui consiste à enjoliver les cultures africaines et dans la moindre mesure celle du peuple Toura ne saurait prospérer longuement. S'il est vrai que la culture africaine regorge d'un potentiel (Éla 1998) incroyable, il est aussi vrai que certains aspects en constituent une faiblesse. Le maintien des aspects négatifs de cette culture est incompatible avec le souci de renforcer la responsabilité individuelle et collective dans un processus de développement local. Dans ce contexte, débarrasser la culture de ses insuffisances sonne comme un défi. Toutefois, il serait illusoire de croire à la facilité d'une telle entreprise. En effet, les changements au niveau culturel sont les plus longs et difficiles à réaliser car ils portent le plus souvent, comme c'est le cas en pays Toura, sur des valeurs profondément enracinées dans la mentalité collective. Normes sociales de représentation, elles constituent l'essence même de l'individu, à savoir son identité. Cela est un fait. Toutefois, le nouvel environnement qui s'impose aux acteurs traditionnels, exige des changements au niveau de certains aspects du rapport entre les individus.

Apporter un changement dans le dualisme respect - crainte des anciens L'élément caractéristique de la société Toura est le respect (Holas 1962 ; Soumahoro 2003) et la peur des anciens (Soumahoro 2003). Des aspects culturels dont font très peu cas les études de développement sur le milieu rural. Il s’agit de phénomènes dont les conséquences sur les paysans ne peuvent être quantifiées. Ces phénomènes ne peuvent, ainsi, se prêter à des analyses statistiques, mais ils demeurent réels. Le paradoxe ici réside dans 
l'ambiguïté qu'il y a entre le respect et la crainte des anciens. On ne sait jamais où le respect s'arrête et où commence la crainte. L'interface entre le respect et la crainte reste élastique et source d'ambiguïté entraînant souvent une inaction de la part de certains acteurs. Le problème est de pouvoir faire une dissociation nette entre le respect, qui relève des rapports sociaux, et la crainte qui relève des considérations plutôt magiques généralement associées au maléfique. Le pouvoir magique ou surnaturel, attribué à tort ou à raison aux anciens, biaise considérablement les rapports sociaux et constitue une sorte d'inertie qui freine les initiatives individuelles. La peur d'une malédiction lorsqu'une initiative est prise de manière isolée et sans l'accord ou l'appui d'un ancien a été plusieurs fois soulignée lors de notre séjour dans les villages. À ce niveau, il doit s'opérer un changement susceptible de libérer les énergies des individus et favoriser le goût de l'action. Nous n'avons pas de solutions à proposer quant à la forme ou au mécanisme qui peut amener ce changement, mais il constitue, au regard de la situation observée sur le terrain, une condition essentielle pour que le développement soit une réalité.

\section{Favoriser un changement dans la rigidité des rapports entre catégories sociales}

Les relations entre les catégories sociales (anciens, jeunes, hommes, et femmes) sont marquées par une rigidité qui laisse très peu de place à un partenariat entre elles dans des activités de développement. Les interfaces d'activité et d'action de développement sont souvent très limitées. La division sociale du travail reste prégnante. Cette division, loin d'être une mauvaise chose en soi, pêche, cependant, par une absence de souplesse qui ne permet pas de jeter un pont et renforcer ainsi l'esprit d'équipe qu'exige une action de développement communautaire. Tout se passe comme si dans l'esprit du villageois Toura les catégories sociales étaient des compartiments étanches ayant des tâches et des fonctions spécifiques. Les propos du genre : «C'est une affaire de femme..., c'est une affaire d'homme..., c'est une affaire des anciens ou des jeunes » lorsqu'il s'agissait de réaliser une activité spécifique que nous avons entendu, lors de l'enquête terrain, sont assez évocateurs de la rigidité des rapports entre les différentes catégories sociales. Cette rigidité est de nature à confiner une catégorie sociale dans des rôles spécifiques mais surtout, et c'est le plus important, à nier volontairement ou involontairement sa capacité d'investir d'autres champs d'action et de compétence. Celle-ci laisse peu de place à l'émergence d'autres valeurs et favorise très peu la dynamique sociale. Il s'agit de rompre avec cette rigidité des rapports entre les différentes catégories sociales afin de créer une dynamique globale qui peut faire appel à toutes les compétences sans exclusivité dans le processus de développement. 
Rompre avec la peur de l'échec et de la réprobation collective La peur de l'échec et de la réprobation collective constitue des valeurs sociales et culturelles de la plupart des paysans Toura. La routine et le statu quo sont, en effet, les fruits de l'ordre social immuable et de son respect par tous. Un processus de passivité sociale s'installe et se traduit non seulement par une absence d'entrepreneuriat, mais aussi par la peur de l'échec et de la réaction du reste de la communauté villageoise. Une tentative effectuée par un paysan est vue par le reste de la communauté comme un défi à l'ordre établi, à tel point que le téméraire finit par désarmer, aidé en cela par les pressions qui ne manquent pas de s'abattre sur lui. La réprobation collective ne se fait, certes, plus de manière frontale, mais sous d'autres formes plus insidieuses comme des conseils répétés, avec arguments à l'appui pour dissuader le paysan d'aller plus loin, soit par des actes de magie ou de sorcellerie, soit par une mise en quarantaine partielle. Le résultat escompté est, certes, le maintien du statu quo, mais aussi un rééquilibrage constant de la structure sociale et un nivelage social pour éviter une émergence marquée d'acteurs susceptibles de bousculer l'ordre établi. La réprobation collective nuit considérablement à l'initiative individuelle et freine l'émergence de paysans innovateurs. Pour que le paysan prenne des initiatives au niveau individuel et qu'il ait le goût du risque, il faudra considérablement atténuer cette pratique.

\section{Participation de tous aux changements}

En dépit des insuffisances soulignées ci-dessus, les conditions d'un développement commandent d'établir une relation de confiance et de compréhension entre les collectivités locales et les intervenants extérieurs. Cette relation doit amener à long terme à une participation de tous aux changements voulus par l'ensemble de la communauté et ce, à plusieurs niveaux d'intervention possible.

La participation des acteurs locaux aux changements exige une participation effective à la prise de décision. Si cet aspect constitue, déjà, une étape importante, il demeure insuffisant. Ce qui est le plus important est que les acteurs aient les moyens de rendre concrets les décisions et les besoins qu'ils ont définis. Les acteurs en ont-ils les moyens et les capacités ? Pour cela, il faut que le paysan soit moins dépendant de l'extérieur dans la réalisation des projets, c'est-à-dire qu'il soit autonome. Toutefois, l'autonomie n'a de substance que par rapport à la capacité des individus et des collectivités à générer de la richesse. Cette dimension essentielle paraît au regard du potentielle socio-économique du pays Toura réalisable avec un minimum d'organisation des acteurs et un certain appui extérieur.

Les paysans et les associations villageoises, au-delà des contraintes d'autonomie, doivent renforcer leurs capacités d'entreprendre pour être plus 
aptes à s'impliquer dans les actions de développement. La valorisation de l'entrepreneuriat villageois doit déboucher sur une relative autonomie financière. Cette approche préconisée ne signifie en aucune manière que l'entrepreneuriat n'existe pas dans la société Toura. Mais, face aux nouveaux défis auxquels les paysans et les associations sont confrontés, il importe pour eux d'apporter des changements à leurs manières d'entreprendre, de devenir plus agressifs, de prendre plus d'initiatives et surtout plus de risques. La prise du risque n'étant pas une habitude paysanne, elle l'est encore moins pour les associations villageoises. Lorsque la certitude et la sécurité d'un acte ne sont pas totalement garanties, le paysan hésite à s'engager. Or le goût du risque est considéré comme l'une des qualités nécessaires à l'entrepreneuriat. Cette qualité n’est pas suffisamment développée tant au niveau individuel qu'au niveau communautaire et collectif. Cette insuffisance explique en partie la faiblesse des actions de développement et le très faible degré de participation.

La participation au développement exige, en même temps, le maintien de la cohésion sociale, une libération des principales forces productives, les jeunes et les femmes, et l'aménagement de nouveaux rapports économiques de ces groupes avec le reste de la collectivité. Il faudra donc s'assurer qu'une initiative locale dans un cadre non-traditionnel, par exemple, un regroupement de jeunes producteurs, obtienne l'assentiment du conseil du village et qu'elle s'exerce en complémentarité des activités traditionnelles et que les retombées d'une telle activité soient clairement perçues par l'ensemble des villageois.

\section{Les stratégies de développement local}

La marmite commence à bouillir par le bas (le fond).

Proverbe Toura

L'élément clé de la stratégie doit être une prise en main effective du développement par les collectivités locales. Au regard de la situation globale du pays Toura, des données sociologiques, écologiques et économiques, il importe de consacrer les efforts de développement sur les besoins internes. L’alternative proposée consiste plutôt à développer la capacité de production dans le but de répondre aux besoins réels de consommation, d'améliorer la qualité de vie et de contribuer au développement régional et ce, grâce entre autres à la vulgarisation de techniques de production plus efficaces, l'organisation de services d'appui à la production agricole et 1'expérimentation de modes de participation autonome des populations locales et de groupes de producteurs innovateurs. Bref, une option qui tient compte des activités, des attitudes et des aspirations de la population régionale et qui veut préserver et 
accroître la capacité d'initiative, de façon à assurer que le développement soit, comme il se doit, le produit d'une participation active et consentie.

Les changements majeurs qui peuvent s'opérer au niveau de la production agricole ou de la diversification des activités doivent, selon nous, passer par la consolidation des activités existantes. Il faudra promouvoir la production agricole de subsistance afin que l'état d'autosuffisance alimentaire que connaît la région demeure et qu'ensuite cette agriculture de subsistance puisse être un appoint à l'agriculture de rente.

Il est de notoriété que l'intervention extérieure a été relativement limitée en pays Toura. Dans ce cas, l'apport extérieur, en tenant compte du niveau actuel de développement, doit s'appuyer sur des technologies appropriées, d'apprentissage simple et de vulgarisation accessible à la grande majorité de personnes. L'objectif à court terme étant une certaine autonomie et flexibilité des acteurs locaux à s'approprier facilement ces technologies, susceptibles d'éviter des coûts excessifs d'apport extérieur en entretien et réparation. Cette approche aura l'avantage de concilier un savoir extérieur moderne avec un savoir faire local offrant moins de contrainte aux acteurs locaux.

Afin d'assurer une meilleure intégration à la réalité sociale et culturelle de la région, le rythme d'intervention devrait s'articuler autour d'un calendrier : 1. d'activités réalisables immédiatement par les villageois sans nécessairement avoir besoin d'appui extérieur. Ce sont généralement des actions simples et peu coûteuses (structure de coordination des associations villageoises, appui à l'action féminine, mise en place d'une comptabilité souple, etc.) 2. d'activités réalisables à court terme par les villageois eux-mêmes ou avec un minimum d’appui extérieur. Ce sont généralement des actions simples et qui demandent peu de moyens venant de l'extérieur (caisse d'épargne et de crédit, développer les cultures maraîchères etc.) 3. d'activités réalisables à moyen terme impliquant aussi bien les acteurs locaux que les acteurs externes. À ce stade, le rôle des acteurs externes dans le soutien aux initiatives locales est important. Il porterait sur l'encadrement et la formation et sur l'appui logistique, humain et budgétaire. 4. d'activités réalisables à long terme. Elles se présentent comme une suite logique des actions antérieures ou du moins leurs conséquences. Elles sont initiées autant par les acteurs locaux que par les acteurs externes (plan directeur du village, intégration des jeunes et femmes au sein du conseil du village etc.).

L'échec ou le succès d’une stratégie de développement dépend énormément de la mobilisation des personnes engagées dans le processus et de leur apport. Ici, il s’agit des paysans Toura. Leur évaluation des besoins est généralement économique, c'est-à-dire qu'avant tout, l'enjeu réside dans la satisfaction des besoins de subsistance. Si la possibilité qu'ils soient associés à une prise de décision est réelle, ils auront la possibilité d'éliminer eux- 
mêmes les obstacles culturels ou sociaux que peut représenter l'introduction de techniques, de matériaux ou de pratiques jugées non conformes aux normes sociales.

Le conseil du village, organe de régulation de la vie sociale au sein de laquelle siège les chefs de famille, a un rôle essentiel à jouer dans le processus de développement. L'appropriation collective et pleine d'une activité ou d'un projet découle de son implication effective et de sa flexibilité à faire accepter aux autres membres de la communauté, si cela est nécessaire, des changements dans les pratiques et lois traditionnelles.

Les producteurs «modernes » constituent dans les villages, au même titre que les cadres en ville, des acteurs nouveaux sur la scène du développement. Leurs activités et surtout leur capacité à s'adapter et à essayer de nouvelles choses font d'eux les possibles catalyseurs du changement dans le milieu local.

\section{Conclusion}

Que devrions-nous retenir de tout ceci ? Une seule chose qui nous paraît essentielle à savoir que la rhétorique qui sous-tend le slogan «Que les populations se prennent en main » bute le plus souvent sur des réalités plus complexes. En effet, concevoir le développement n’est pas chose aisée, encore moins lorsqu'il s'agit de concevoir le développement des autres à leur place sans nécessairement les associer comme cela a été longuement pratiqué dans le milieu rural africain. Un tel dessein serait en lui-même contradictoire. Le développement ou encore l'évolution autonome à travers un processus de développement endogène et local d'une société, ne relève pas de la planification ou de l'ingénierie, mais procède d'un projet social réalisé selon les attentes des collectivités concernées, avec leur accord et leur participation active. Il comporte fatalement une finalité et en cela il est intimement lié à la société qui l'incarne.

Que retenir de la société Toura ? Qu'elle est dans une phase de mutation toujours marquée par un socle traditionnel très fort symbolisé par le conseil du village. Les signes de fléchissement font apparaître progressivement une tendance à l'intégration de l'apport extérieur dans les pratiques et modes de gestion socio-économique. Les associations de développement constituent l'épicentre de la transformation sociale de l'individu et le fondement des initiatives de développement. Toutefois ces associations se heurtent encore aux structures traditionnelles que des années de modernisation ne suffiront pas à faire disparaître facilement.

Tout compte fait, les nouveaux besoins et aspirations nés d'un modèle de développement imposé sont toujours d'actualité car ils n'ont pas été satisfaits. Les indices d'un sous-développement rural sont présents. En effet, le 
processus de modernisation certes embryonnaire n'a pas encore atteint toutes les localités. L'émergence des acteurs et paysans modernes reste encore fragmentaire et la volonté de développement n'est pas non plus affichée avec détermination par tous les acteurs.

Le développement n'étant pas une affaire d'expert, telle est notre intime conviction, il faut voir dans les conditions et les stratégies de développement évoquées plus haut, non pas une recette de développement prête à être exécutée, mais des grandes pistes d'action susceptibles de créer les conditions d'un développement véritable, donc autonome et en conformité avec les aspirations des populations.

\section{Notes}

1. La recherche terrain dans le cadre de cette étude a été faite en 2001 avant le déclenchement en 2002 du conflit armé que connaît actuellement la Côte d'Ivoire. L'analyse qui est faite dans cette étude tient donc compte du contexte antérieur à la crise même s'il convient d'admettre que la situation actuelle de crise que vit la Côte d'Ivoire et surtout la région Ouest renforce encore plus la nécessité de prendre en compte l'approche préconisée dans cet article et que la problématique posée est plus que jamais d'actualité.

\section{Références}

Aubin, B., 1991, Aménagement des terroirs et participation populaire : Éléments du débat sur les approches participatives, Centre Sahel, Université Laval, février, Série Dossiers n ${ }^{\circ} 15$.

Banque mondiale, 1996, Rapport annuel. Principaux programmes de la Banque mondiale : exercice 96. (http://www.worldbank.org/html/extpb/annrep96/ french/contents.htm). 25 février 2006.

Beaudoux, E., 2001, Accompagnement des acteurs locaux à la gestion et l'entretien des investissements : outils et propositions, Hydro-Pacte, Iram (Institut de recherche et d'application des méthodes de développement).

Belloncle, G., 1982, La question paysanne en Afrique noire, Paris:Karthala, 110 p. Bernier, J., 1984, « Problématique de développement de la région de Bolama (GuinéeBissau) », Culture et développement, Université Catholique de Louvain, Vol.16, $195 \mathrm{p}$.

Bernier, J., 1988, « Plaidoyers populistes en faveur de la paysannerie africaine », Canadian Journal of African studies/ Revue canadienne des études africaines, Toronto, Volume 20, p. 441-444.

Boiral, P., Lanterre, J. F., Olivier de Sardan, J.-P., dir., 1985, Paysans, experts et chercheurs en Afrique noire. Sciences sociales et développement rural, Paris :Ciface-Karthala, $224 \mathrm{p}$.

Dupriez, H., 1985, Paysans d’Afrique noire, 3e éd., Paris:L’Harmattan, , 253 p. 
Ela, J. M., 1998, Innovations sociales et renaissance de l'Afrique noire, Paris:Harmattan, $425 \mathrm{p}$.

Éla, J. M., 1982, L’Afrique des villages, Paris:Karthala.

FAO, 2004, Les approches de développement centrées sur les gens. Programme d'Appui aux moyens d'Existence (LSP), Un Programme interdépartemental pour l'amélioration de l'appui aux moyens d'existence de la population rurale pauvre, Document de Travail, FAO, Rome, 73 p.

Gonin, G., 1986, Rapports entre Mandé et peuples forestiers et préforestiers de l'ouest de la Côte d'Ivoire à travers les traditions orales Tura (Milieu du XVIIe siècle - début XXe siècle).Thèse de Doctorat de 3e cycle, Université de Paris I Panthéon-Sorbonne, 363 p.

Hauhouot, A., Kobi, A., Koffi A., Zamblé, B. I., 1992, Populations, traditions et développement dans la région Ouest de la Côte d'ivoire : Le cas de la souspréfecture de Man, Université Nationale de Côte d'Ivoire, Institut de géographie tropicale (IGT), $171 \mathrm{p}$.

Haut Commissariat au Développement Intégré de la Région Semi-Montagneuse de l'Ouest (HCDI_RSMO), 1998, Schéma Directeur pour le développement intégré de la région semi-montagneuse de l'Ouest, $200 \mathrm{p}$.

Hochet, A., N'Gar, A., 1995, Développement rural et méthodes participatives en Afrique, Paris: L’Harmattan, 208 p.

Holas, B., 1962, Les Toura, Esquisse d'une Civilisation Montagnarde de Côte d'Ivoire, Paris :PUF, 334 p.

Lévy, M., 2000, "Comment renouveler les politiques de coopération au développement ? », Esprit, nº 6, pp.79-100.

Meister, A., 1977, La participation pour le développement. Paris:Éditions Ouvrières, 176 p.

Mendras, H., 1991, La fin des paysans, Coédition Actes-sud-labor-l'aire, 433 p.

Ouédraogo, B., 1990, Entraide villageoise et développement. Groupements paysans au Burkina Faso, Paris : L’Harmattan, 177 p.

Pradervand, P., 1989, Une Afrique en marche-la révolution silencieuse des paysans africains, Paris:Plon.

Prevost, 2001, Le développement local : contexte et définition, cahiers de recherche IREC 01-03, IRECUS, Université de Sherbrooke, 28 p.

Soumahoro, M., 2003, Activité humaine et développement durable dans la région montagneuse de l'ouest de la Côte d'Ivoire : le cas du pays Toura, Thèse de doctorat, Université Laval, Département de géographie, 506 p. 